\title{
IAMJ
}

INTERNATIONAL

AYURVEDIC

MEDICAL JOURNAL

Review Article

ISSN: 2320-5091

Impact Factor: 6.719

\section{MANA IN BHAISHAJYA KALPANA: A CRITICAL REVIEW}

\author{
Rachana Sharma $^{1}$, Govind Sahay Shukla ${ }^{2}$, Rajaram Agarwal ${ }^{3}$, Manisha Goyal $^{4}$ \\ ${ }^{1}$ M.D. Scholar (P.G. Department of Rasa shastra and Bhaishajya Kalpana), \\ ${ }^{2}$ Prof. \& H.O.D. (P.G. Department of Rasa shastra and Bhaishajya Kalpana), \\ ${ }^{3}$ Associate. Prof. (P.G. Department of Rasa shastra and Bhaishajya Kalpana), \\ ${ }^{4}$ Associate. Prof. (P.G. Department of Rasa shastra and Bhaishajya Kalpana), \\ Dr. Sarvapalli Radhakrishnan Rajasthan Ayurveda University, Karwar, Jodhpur, Rajasthan, India
}

Corresponding Author: rachanasharma040893@gmail.com

\section{https://doi.org/10.46607/iamj2109112021}

(Published Online: November 2021)

Open Access

(C) International Ayurvedic Medical Journal, India

Article Received: 17/10//2021 - Peer Reviewed: 30/10/2021 - Accepted for Publication: 31/10/2021

\section{Check for updates}

\section{ABSTRACT}

The utility of weights and measures is an integral part of today's life. From any part of human history, the indirect or direct clues for the use of measuring units can be readily traced. This system of measurements is considered a basic principle when it comes to the study of any sciences. Rasashastra and Bhaishajya Kalpana in Ayurveda deal with studies on medicine dosage and administration in the prescribed manner. In ancient Mana methods of Magadha Mana and Kalinga Mana are compared in this article. Pautavamana, Druvayamana, and Payyamana are three types of mana based on the nature of the substance that is highly useful in Aushadha sevan matra. Therefore, for all purposes (pharmaceutical as well as therapeutic) the consideration of 'Mana' is essential. Because of the greater significance laid upon 'Mana' in the field of medicine.

Keywords: Mana, Magadha mana, Kalinga mana, Pautavamana, Druvayamana, Payyaymana. 


\section{INTRODUCTION}

Mana is an inseparable part of our knowledge and studies. Without the knowledge of 'Mana' one can never expect to have a thorough approach to any subject matter. Mana is defined as the system of measurement, based on the measures of weight, length, or capacity. Nowadays M.K.S or C.G.S or F.P.S method is used for measurement for a different form. Depending on the area in which the systems of measurements prevailed, they are classified as - 1 . Magadha mana (Followed by Acharya Charaka) 2. Kalinga mana (Followed by Acharya Sushruta) In Amara kosha, Mana was classified as Pautavmana, Druvayamana, Payyamana. This mana indicates the weight, volume and lengthwise measurement respectively. According to British Pharmacopia: 1. Imperial System, 2. Metric System Mana is an inseparable part of our knowledge and studies. Without the knowledge of 'Mana', one can never expect to have a thorough approach to any subject matter.

- To study measurement in our classics

- It is inseparable and most essential in all eight branches of Ayurveda.

- Plays a crucial role in quadruples of treatment.

- For quantitative assessment of body constituents.

- Raw drug collection

- Preparation of different Kalpana's

- Anupana matra
- Preparation of Ahara kalpana (Pathya kalpana)

- To quantity the drugs for all purposes.

- To fix the doses etc.

- According to Acharya Agnivesha Mana, is considered for the following things: Dosha, Bala, Satmya, Bheshaja, Sharira, Satwa, Desh, Sara, Prakruti, Kala, Ahara, Vaya etc.

Differences in Magadha mana and Kalinga mana: -

1. Acharya Sharangdhar considers Magadha mana as superior to the Kalinga mana.

2. Magadha mana prevailed in Maurya dynasty in Magadha desha, and Kalinga mana prevailed in Kalinga desha which was ruled by Kalinga kings.

3. Magadha mana was followed by Maharshi Charaka and Kalinga mana by Maharshi Sushruta.

4. In Magadha mana 6 Gunja will be 1 Masha (750 gm) whereas in Kalinga mana 8Gunja will be 1 Masha (1000 mg or $1 \mathrm{gm}$ )

5. Amarkosha- Depending on the nature of the measuring material, the Mana is classified as Pautava mana- To measure the weight of solid content, Druvayamana- To measure the quantity of liquid, Payyamana- To measure the length of the herbal drugs, medical instruments, patient height, pharmacy measurements etc.

\section{Magadha mana: -}

(a) Acco. to Acharya Sharangdhar: -

\begin{tabular}{|l|l|l|}
\hline 30 Parmanu & 1 Vanshi & \\
\hline 6 Vanshi & 1 Marichi & \\
\hline 6 Marichi & 1 Rajika & \\
\hline 3 Rajika & 1 Sarshapa & $125 \mathrm{mg}$ \\
\hline 8 Sarshapa & 1 Yava & $750 \mathrm{mg}$ \\
\hline 4 Yava & 1 Gunja (Ratti/Rakti) & $3 \mathrm{gm}$ \\
\hline 6 Gunja & 1 Masha (Rajmasha, Hem, Dhanyak) & $6 \mathrm{gm}$ \\
\hline 4 Masha & 1 Shana (Tank, Dharan) & $12 \mathrm{gm}$ \\
\hline 2 Shana & 1 Kola (Kshudrak, Vataka, Drakshana) & $24 \mathrm{gm}$ \\
\hline Kola & $\begin{array}{l}\text { 1 Karsha (Panimanika, Aksha, Akshapichu, Panitala, Kinchitpani, Tinduka, } \\
\text { Vidalpadaka, Shodashika, Karmadhya, Hanspada, Suvarna) }\end{array}$ & 48gm \\
\hline 2 Karsha & 1 Shukti (Ashtmika, Ashtamansha) & \\
\hline 2 Shukti & 1 Pala (Amra, Mushti, Chaturthika, Prakuncha, Shodashi, Bilva & \\
\hline
\end{tabular}




\begin{tabular}{|l|l|l|}
\hline 2 Pala & 1 Prasrata (Ardhanjali) & $96 \mathrm{gm}$ \\
\hline 2 Prasrata & 1 Kudava (Anjali, Ardha saravaka, Ashtamana, Ashtashukti) & $192 \mathrm{gm}$ \\
\hline 2 Kudava & 1 Manika (Sarava) & $384 \mathrm{gm}$ \\
\hline 2 Manika & 1 Prastha & $768 \mathrm{gm}$ \\
\hline 4 Prastha & 1 Adhaka (Bhajan, Kanspatra) & $3.072 \mathrm{~kg}$ \\
\hline 4 Adhaka & 1 Drona (Kalansh, Nalvana, Unmana, Ghta, Rashi) & $12.228 \mathrm{~kg}$ \\
\hline 2 Drona & 1 Shurpa (Kumbha) & $24.576 \mathrm{~kg}$ \\
\hline 2 Shurpa & 1Droni (Vahi, Goni) & $49.152 \mathrm{~kg}$ \\
\hline $\begin{array}{l}\text { 4 Droni } \\
\text { 2000 } \\
\text { (20Tula) }\end{array}$ & 1 Khari & $196.608 \mathrm{~kg}$ \\
\hline 100 Pala & 1 Bhara & $96 \mathrm{~kg}$ \\
\hline
\end{tabular}

(b)Acco. to Acharya Charaka: -

\begin{tabular}{|l|l|}
\hline 6 Dhwanshi & 1 Marichi \\
\hline 6 Marichi & 1 Raktsarshapa \\
\hline 8 Raktsarshapa & 1 Tandula \\
\hline 2 Tandula & 1 Dhanyamash \\
\hline 2 Dhanyamash & 1 Yava \\
\hline 4 Yava & 1 Andika \\
\hline 4 Andika & 1 Masha (Hem, Dhanyak) \\
\hline 3 Masha & 1 Shana \\
\hline 2 Shana & 1 Drankshan (Kol, Badar) \\
\hline 2 Drankshan & 1 Karsha (Suvarna Aksha, Vidalpadaka, Pichu, Panitala, Tinduka, Kavalgrah) \\
\hline 2 Suvarna & 1 Palardh (Shukti, Ashtmika) \\
\hline 2 Palardh & 1 Pala (Mushti, Prakunch, Chaturthika, Bilva, Shodashika, Amra) \\
\hline 2 Pala & 1 anjali (kudava) \\
\hline 2 Kudava & 1 manika \\
\hline 4 Kudava & 1 prastha \\
\hline 4 Prastha & 1 aadhaka (kansa) \\
\hline 4 Kansa & 1 drona (armana, nalvana, kalash, ghata, unmana) \\
\hline 2 Ghata (Drona) & 1 Shurpa \\
\hline 2 Shurpa & 1 Goni (Khari, Bhara) \\
\hline 32 Shurpa & 1 Vaah \\
\hline 100 Pala & 1 Tula \\
\hline
\end{tabular}

(c)According to Acharya Sushruta: -

\begin{tabular}{|l|l|}
\hline 12 Dhanyamash & 1 Suvarnamash (1 Masha) \\
\hline 16 Suvarnamash & 1 Karsha (Suvarna 1 Tola) \\
\hline 19 Nishpav & 1Dharan \\
\hline 2.5 Dharan & 1 Karsha $($ Tola $)$ \\
\hline 4 Karsha & 1 Pal $(4$ Tola) \\
\hline 4 Pala & 1 Kudawa $(3$ Chatank 1 Tola) \\
\hline 4Kudawa & 1 Prastha $(12$ Chatank 4 Tola) \\
\hline 4 Prastha & 1 Aadhaka $(3$ Ser 3 Chatank 1 Tola) \\
\hline 4 Aadhak & 1 Drona $(12$ Sera 12 Chatank 4 Tola) \\
\hline 400 Karsha & 1 Tula $(5$ ser $)$ \\
\hline 20 Tula & 1 Bhar $(100$ ser $)$ \\
\hline
\end{tabular}




\section{Unit of Mana in classics texts}

\begin{tabular}{|c|c|c|c|c|}
\hline S.NO. & MANA & CHARAKA & SHARANGDHAR & SUSHRUTA \\
\hline 1 & Parmanu & - & + & _ \\
\hline 2 & Vanshi (Dhawanshi) & + & + & - \\
\hline 3 & Marichi & + & + & - \\
\hline 4 & Rajika & & + & - \\
\hline 5 & Sarshapa & + & + & - \\
\hline 6 & Tandula & + & & - \\
\hline 7 & Dhanyamasha & + & & - \\
\hline 8 & Yava & + & & _ \\
\hline 9 & Andika & + & _- & - \\
\hline 10 & Gunja (Ratti/Rakti) & + & + & _ \\
\hline 11 & Masha (Hem, Dhanyak) & + & + & + \\
\hline 12 & Shana (Tank, Dharan) & + & + & + \\
\hline 13 & Kola (Drankshan, Kol, Badar) & + & + & - \\
\hline 14 & $\begin{array}{l}\text { Karsha (Panimanika, Aksha, } \\
\text { Akshapichu, Panitala, Kinchitpani, } \\
\text { Tinduka, Vidalpadaka, Shodashika, } \\
\text { Karmadhya, Hanspada, Suvarna) }\end{array}$ & + & + & + \\
\hline 15 & Shukti (Ashtmika, Ashtamansha) & $+($ palardh $)$ & + & - \\
\hline 16 & $\begin{array}{l}\text { Pala (Amra, Mushti, Chaturthika, } \\
\text { Prakuncha, Shodashi, Bilva) }\end{array}$ & & & + \\
\hline 17 & Prasarata (Ardhanjali) & + & + & _ \\
\hline 18 & $\begin{array}{l}\text { Kudawa (Anjali, Ardha Saravaka, } \\
\text { Ashtamana, Ashtashukti) }\end{array}$ & + & + & + \\
\hline 19 & Manika (Sarava) & + & & - \\
\hline 20 & Prastha & + & & $\overline{+}$ \\
\hline 21 & Aadhaka (Bhajan, Kanspatra) & & & + \\
\hline 22 & $\begin{array}{l}\text { Drona (Kalansh, Nalvana, Unmana, } \\
\text { Ghta, Rashi) }\end{array}$ & & & + \\
\hline 23 & Shurpa (Kumbha) & & & _ \\
\hline 24 & Droni (Vahi, Goni) & + & & - \\
\hline 25 & Goni (Khari, Bhara) & + & + & + \\
\hline 26 & Vaah & + & _ & _- \\
\hline 27 & Tula & + & + & + \\
\hline
\end{tabular}

\section{Chaturguna niyam by Acharya Sharangdhara}

\begin{tabular}{|c|c|c|c|}
\hline Mana & Equivalent & Metric equivalent & Synonyms \\
\hline 4 Masha & 1 Tanka & $3 \mathrm{gm}$ & Shana, Dharana \\
\hline 4 Tanka & l Aksha & $12 \mathrm{gm}$ & $\begin{array}{l}\text { Karsha, Panimanika, Akshpichu, Paniatala, Kinchitpani, Tinduka, } \\
\text { Vidalpadaka, Shodashika, Karamadhya, Hanspada, Suvarna }\end{array}$ \\
\hline 4 Aksha & l Bilva & $48 \mathrm{gm}$ & Pala, Mushtiamra, Chaturthika, Prakuncha, Shodashi \\
\hline 4 Bilva & l Kudava & $192 \mathrm{gm}$ & Anjali, Ardhsaravaka, Ashtashukti \\
\hline 4 Kudava & 1 Prastha & $768 \mathrm{gm}$ & \\
\hline 4 Prastha & 1 Aadhaka & $3 \mathrm{~kg}$ & Bhajan, Kanspatra \\
\hline 4 Aadhaka & 1 Rashi & $12.288 \mathrm{~kg}$ & Drona, Kalansh, Nalvana, Unmana, Ghata \\
\hline 4 Rashi & 1 Droni & $49.152 \mathrm{~kg}$ & Vahi, Goni \\
\hline 4 Droni & 1 Khari & $196.6 \mathrm{~kg}$ & \\
\hline
\end{tabular}




\section{Kalinga Mana: -}

\begin{tabular}{|c|c|c|}
\hline 12 Gaursarshapa & 1 Yava & \\
\hline 2 Yava & 1 Gunja & $125 \mathrm{mg}$ \\
\hline 3 Gunja & 1 Valla & $375 \mathrm{mg}$ \\
\hline 8 Gunja & 1 Masha $($ Acco to some 7 Gunja $=1$ Masha $)$ & $1000 \mathrm{mg}$ or $1 \mathrm{gm}$ \\
\hline 4 Masha & 1Shana & $4 \mathrm{gm}$ \\
\hline 6 Masha & 1 Gadhyan & $6 \mathrm{gm}$ \\
\hline 10 Masha & 1 Karsha & $10 \mathrm{gm}$ \\
\hline 4 Karsha & 1 Pala (10 shana) & $40 \mathrm{gm}$ \\
\hline 4 Pala & 1 Kudava & $160 \mathrm{gm}$ \\
\hline
\end{tabular}

1. Pautavamana (Measurement of weight)- Rasvagbhatokta Pautavamana chart: -

\begin{tabular}{|c|c|}
\hline 6 Anu & 1 Truti \\
\hline 6 Truti & 1 Liksha \\
\hline 6 Liksha & 1 Yuka \\
\hline $6 Y u k a$ & 1 Raja \\
\hline 6 Raja & 1 Sarshap \\
\hline 6 Sarshap & 1 Yava \\
\hline 6 Yava & 1 Gunja \\
\hline 2 Gunja & 1 Nishpav \\
\hline 3 Gunja & 1 Valla \\
\hline 2 Valla & 1 Masha \\
\hline 2 Masha & 1 Dharan \\
\hline 2 Dharan & 1 Shan (nishka, kala) \\
\hline 2 Nishka & 1 Vataka(kola) \\
\hline 2 Kola & 1 Tola (Karsha, Nishka, Chatushtya, Udumbar, Panitala, Suvarna, Kavalgraha, Vidalpadaka) \\
\hline 2 Tola & 1 shukti \\
\hline 2 Shukti & 1 pala (as per other opinion 3 shukti-1 pala)(Mushti, prakunch, vilba) \\
\hline 2 Pala & 1 prasarta \\
\hline 2 Prasrata & 1 kudava (1 anjali) \\
\hline 2 Kudava & 1 manika \\
\hline 2 Manika & 1 prastha \\
\hline 2 Prastha & 1 shubh \\
\hline 2 Shubh & 1 patraka (aadhak, bhajan, patra) \\
\hline 4 Aadhak & 1 drona (ghta, unmana, nalvana, arman, kumbhak) \\
\hline 100 Pala & 1 tula \\
\hline 4000 Pala/40 Tula & 1 bhara \\
\hline
\end{tabular}

\section{Bhartiya Pautavamana: -}

\begin{tabular}{|l|l|}
\hline 6 Ratti & 1Ana \\
\hline 24 Ratti & 4 Ana \\
\hline 48 Ratti & 8 Ana \\
\hline 96 Ratti & 1 Rupee \\
\hline 5 Tola & 1 Chatanka \\
\hline 4 Chatanka & 1 Pava \\
\hline 8 Chatanka & 0.5 Sera \\
\hline 16 Chatanka & 1 Sera \\
\hline 40 Sera & 1 Man \\
\hline 27 An & 1 Tan \\
\hline
\end{tabular}


2. Druvayamana (Measurement of liquid)- Druvayamana is the measure of capacity. The smallest unit here is 'Bindu'(drop). One Bindu is a drop of liquid that falls from the index finger after it is lifted from the water.

According to Ashtang Hridya

\begin{tabular}{l|l}
\hline 8 Bindu & 1 Shana (16 drops will make $1 \mathrm{ml})$ \\
\hline 32 Bindu & 1 Shukti \\
\hline 64 Bindu & 1 Panishukti \\
\hline
\end{tabular}

3. Payyamana (Measures of length)- One Angula is said to be the basic unit here, explained as the length of 8 Yava brought together in one thread or placed one besides the other.

\begin{tabular}{|l|l|l|}
\hline Classical units & Inches & Metric equivalents \\
\hline Yavodara & $1 / 8$ or $3 / 4$ inch & $0.24 \mathrm{~cm}$ \\
\hline Angula & $3 / 4$ inch & $1.95 \mathrm{~cm}$ \\
\hline Vitasti & 9 inches & $22.86 \mathrm{~cm}$ \\
\hline Aratni & $101 / 2$ inch & $41.91 \mathrm{~cm}$ \\
\hline Hasta & 18 inches & $45.72 \mathrm{~cm}$ \\
\hline Rajhasta & 22 inches & $55.88 \mathrm{~cm}$ \\
\hline Vyama & 72 inches & $182.88 \mathrm{~cm}$ \\
\hline
\end{tabular}

Pashchatya mana paribhasha (Weight and tem is an old system based on arbitrary and unrelated measures Acco. to Modern): -

There are two systems of weights and measures (a) units. The metric system or decimal system is based Imperial system (b) Metric system. The imperial syson related and rationally derived units

\section{Metric system: -}

\begin{tabular}{|l|l|}
\hline 1 kilogram $(\mathrm{Kg})$ & $1000 \mathrm{gm}$ \\
\hline 1 Hectogram $(\mathrm{Hg})$ & $100 \mathrm{gm}$ \\
\hline 1 Decagram $(\mathrm{deg})$ & $10 \mathrm{gm}$ \\
\hline 1 Gram $(\mathrm{gm})$ & $1 \mathrm{gm}$ \\
\hline 1 Decigram $(\mathrm{dg})$ & $0.1 \mathrm{gm}(100 \mathrm{mg})$ \\
\hline 1 Centigram $(\mathrm{cg})$ & $0.01 \mathrm{gm}(10 \mathrm{mg})$ \\
\hline 1 Milligram $(\mathrm{mg})$ & $0.001 \mathrm{gm}(1 \mathrm{mg})$ \\
\hline 1 Microgram $(\mathrm{mcg})$ & $1 / 1000 \mathrm{mg}$ \\
\hline
\end{tabular}

Domestic measures:

\begin{tabular}{|l|l|l|}
\hline 1 drop & 1 minim & $0.06 \mathrm{ml}$ \\
\hline 1 teaspoonful & $1 \mathrm{fl}$. Drachm & $4 \mathrm{ml}$ \\
\hline 1 dessert spoonful & $2 \mathrm{fl}$. Drachm & $8 \mathrm{ml}$ \\
\hline 1 table spoonful & $4 \mathrm{fl}$. Drachm & $15 \mathrm{ml}$ \\
\hline 2 table spoonsful & 1 fl. Ounce & $30 \mathrm{ml}$ \\
\hline 1 wine glassful & 2 fl. Ounce & $60 \mathrm{ml}$ \\
\hline 1 tea cupful & 4 fl. Ounce & $120 \mathrm{ml}$ \\
\hline 1 tumblerful & 8 fl. Ounce & $240 \mathrm{ml}$ \\
\hline
\end{tabular}


Imperial system: - The imperial system of measurements uses ounces and pounds to measure weight; pint and gallons to measure volume; inches, feet, and yards to measure length. Some of the useful measuring units are below: -

Metric system of weight

10 miligram

10 centigrams

10 decigrams

10 grams

10 decagrams

10 hectograms

$1000 \mathrm{~kg}$

1 centigram
1 decigram
1 gram
1 decagram
1 hectogram $(100 \mathrm{gm})$
1 kilogram
1 metric ton

1/10 litre

1/100 litre

$1 / 1000$ litre

10 litres

100 litres

1000 litre

Metric system of length

\begin{tabular}{|l|l|}
\hline 1 meter & 39.37 inch \\
\hline 1 decimeter & $1 / 10$ meter \\
\hline 1 centimetre & $1 / 100$ meter \\
\hline 1 millimetre & $1 / 1000$ meter \\
\hline 1 decameter & 10 meters \\
\hline 1 hectometer & 100 meters \\
\hline 1 kilometre & 1000 meter \\
\hline
\end{tabular}

Kala mana (Measurement of time): While preparing the different medicines, the time adjustment should be taken into consideration and the time of medicine administration after preparation, preservative time also will be decided to depend upon the $\mathrm{Ka}$ - la (Time). Kala mana, the measuring unit of time is mentioned at different instances in the classics. The basic units include Nimesha (closing eyelids) and Unmesha (opening eyelids) together as one Matra kala.

\begin{tabular}{|l|l|}
\hline 1 Ksana & $4 / 5$ of a sec, an instant, a moment $(0.38 \mathrm{sec})$ \\
\hline 2 Ksana & 1 Lava $\left(1 / 6^{\text {th }}\right.$ of a wink $)(0.77 \mathrm{sec})$ \\
\hline 2 Lava & 1 Nimesha $(1.55 \mathrm{sec})$ \\
\hline 3 Nimesha & 1 Kashtha $\left(1 / 30^{\text {th }}\right.$ of kala $)(4.66 \mathrm{sec})$ \\
\hline 30 Kashtha & 1 Kala $(2 \mathrm{~min} 20 \mathrm{sec})$ \\
\hline 20 Kala +3 Kashtha & 1 Muhurata $(48 \mathrm{~min})$ \\
\hline $1 / 2$ of Muhurata & 1 Ghati $(24 \mathrm{~min})$ \\
\hline 30 Muhurta & 1 Ahoratra $(24$ hrs $)(8$ Yama $)$ \\
\hline 1 Yama or 1 Prahara & 3 hours \\
\hline 15 Ahoratra & 1 Paksha $(15$ days $)$ \\
\hline
\end{tabular}




\begin{tabular}{|l|}
\hline 2 Paksha \\
\hline 2 Mas \\
\hline 3 Ritu \\
\hline 2 Ayana \\
\hline 5 Samvatasara
\end{tabular}

1 Mas (30 days)

1 Ritu (60 days)

1 Ayana (6 months)

1 Samvatsara (12 months)

5 Samvatasara

1 Yuga (5 years)

\section{DISCUSSION}

The utility of weights and measures is an integral part of our day-to-day life. In Ayurveda Mana plays an important role in the preparation of drug formulation to drug intake, Pathya, Anupana etc. This 'system of measurements' is considered as a basic principle when it comes to the study of any sciences. In our classics, different types of Mana are explained: Magadha mana, Kalinga mana, Pautava mana, Payya mana, Druvaya mana, Kala mana etc. Acharya Sharangdhar and Charaka write Magadha mana as superior among both. Acharya Charaka deal with the smallest unit of Magadha mana is 'Dhawanshi' and Sharangdhar smallest unit of Magadha mana is 'Parmanu'. Acco. to Charaka 3 masha is equal to 1 Shana and Acco. to Sharangdhar 4 Masha is equal to 1 Shana, But from Kola to Goni there is no change. Mostly the Pautavamana is used in the reference of Aushadha sevan matra, Aushadha nirman matra etc. Payyamana used in the reference of Dhumpana, Puta, Sharair pramana. Druvayamana used in Avleha kalpana, Nasya kalpana, Aasava-Arishta, Ksheerpa$k a$. Metric equivalents of weights and measures, linear measures and measurement of time used in the Ayurvedic classics have been approved by the Ayurvedic Pharmacopoeia Committee in consultation with the Indian standard institution.

\section{CONCLUSION}

In Bhaishajya Kalpana Mana was considered in the basic principles. Although there is a difference between Mana Paribhasha by Acharyas, the Mana remains the same. Acharya Charaka, Sharangdhara described the two types of Mana, Acharya Amarkosha mentioned three types of mana in their Samhita. According to British Pharmacoepia 2 types of mana are described. Nowadays the Magadha mana men- tioned by Acharya Sharangdhara and Acharya Charaka is being practised.

\section{REFERENCES}

1. DR. Smt. Shailja Shrivastava, Sharangdhar Samhita, Chapter 1 (Manglacharanam), Pratham khand, Chowkhamba orientalia, Varanasi, Edition 2016, Pg. no. 6-8

2. Satyanarayan Shashtri, Charaka Samhita, Chapter 12 (Dantidravanti kalpa adhyay), VOL.-2, Chowkhamba bharti akadami, Varanasi, Reprint edition 2012, Page no. 954

3. Dr Ambikadutt Shastri, Sushruta samhita, commentary- "Ayurvedtatva sandipika", Chapter 39 (Aaturopdrava chikitsa), Chaukhamba Sanskrit Sansthan, Varanasi, Reprint Edition 2018, Page no. 166

4. Dattatreya Anant Kulkarni, Ras ratna samuchya, commentary- "Vigyan Bodhini" Chapter-11 (Rasa paribhasha), Meharchand Lakshmandas publications, Edition, Page no.-203

5. DR. Ravindra Angadi, A textbook of "Bhaishajya kalpana vigyana pharmaceutical science", Chapter-2 (Mana paribhasha), Chaukhamba surbharti prakashan, Varanasi, $2^{\text {nd }}$ revised edition 2016, Page no.18

6. DR. K. Rama Chandra Reddy, Bhaishajya kalpana vigyana, Chapter 5 (Mana-measurements), Chaukhamba Sanskrit bhawan, Varanasi, Fourth edition 2018 Page no. 94

7. The Ayurvedic Formulary of India, (Govt. of India) (Ministry of health and family welfare), Part 3, First edition, Page no-705

8. Shri pandit hargovind shastry, Amarsimha, Amarkosha, Hindi commentary (Sarala-maniprabha), Chowkhambha Sanskrit sansthan, Varanasi, iv ${ }^{\text {th }}$ edition.

9. Acharya Siddhinandan Mishra, Bhaishajya Kalpana Vigyan, Chapter 3 (Mana Paribhasha), Chaukhamba Orientalia, Varanasi, 2014 edition, Page no. 54

10. Dr Indradev Tripathi, Rasarnav Rasatantram, Chapter 10 (Rasa vishyaka prashna), Chaukhambha Amarbharti Publication, Varanasi, $2^{\text {nd }}$ edition, Page no. 135 
11. Shree Ambikadatta Shastri, Shree Govindadas virachita, Bhaishajya Ratnavali, Chaukhambha Sanskrita Sansthana, Varanasi, $14^{\text {th }}$ edition, 2001, page no.1261

12. Pandita Kashinath Shastri, Sadanand Sharma virachita, Rasa tarangini, Chapter 8 (Gandhaka vigyaniya), Uttara khanda, Motilal Banarasidas, Delhi (India), $8^{\text {th }}$ edition, 2014, page no. 249

\section{Source of Support: Nil}

\section{Conflict of Interest: None Declared}

How to cite this URL: Rachana Sharma et al: Mana In Bhaishajya Kalpana: A Critical Review. International Ayurvedic Medical Journal \{online\} 2021 \{cited November 2021\} Available from: http://www.iamj.in/posts/images/upload/2775_2783.pdf 